\title{
LA RÉPARTITION GÉOGRAPHIQUE DES POISSONS D'EAU DOUCE EN FRANCE : QUI EST AUTOCHTONE ET QUI NE L'EST PAS ?
}

\author{
H. PERSAT (1) et P. KEITH (2).
}

(1) ESA CNRS 5023, Ecologie des Eaux Douces et des Grands Fleuves, Bâtiment 403, Université Lyon 1, 69622 Villeurbanne Cedex, France.

(2) Institut d'Ecologie et de Gestion de la Biodiversité, Muséum National d'Histoire Naturelle, 57 rue Cuvier, 75233 Paris Cedex 05, France.

Ministère de l'Environnement, Direction de l'Eau, 20 avenue de Ségur, 75302 Paris 07 SP, France.

\section{RÉSUMÉ}

Les bouleversements intervenus ces dernières décennies dans la composition de l'ichtyofaune d'eau douce française incitent, d'une part, à faire un bilan de la situation présente (cf. l'Atlas des poissons d'eau douce de France) et, d'autre part, à promouvoir une politique de gestion cohérente avec le principe de protection du patrimoine biologique naturel. Cette notion de patrimoine reste toutefois à définir. En effet, les changements actuels ne sont que les derniers d'une longue série, où l'homme est un acteur capital depuis plus longtemps qu'on ne le croit. De fait, beaucoup des espèces dites françaises n'ont probablement pas de valeur patrimoniale dans nombre de nos bassins fluviaux. Le présent article a pour ambition de décrire l'ampleur du problème et de solliciter la communauté scientifique, afin de rassembler les informations et développer les investigations selon un cadre géographique et historique précis.

\section{THE GEOGRAPHIC DISTRIBUTION OF FRESHWATER FISHES IN FRANCE : WHICH ARE NATIVE AND WHICH ARE NOT?}

\section{SUMMARY}

The major changes observed in the French freshwater fish fauna during the last decades incited us, first, to set up the present state (Atlas of the freshwater fishes in France) and, secondly, to promote a management policy in accordance with the protection of the natural heritage. However, this last notion remains to be defined. Indeed, the present changes are the last of a long series, in which man has been a chief actor for a much longer time than usually believed. Consequently, many of the assumed French species have probably no true patrimonial value in many of our fluvial catchments. The aim of the present paper is to show the broad scope of this problem and to appeal to the scientific community for gathering information and developing investigations within an accurate geographical and temporal frame.

\section{INTRODUCTION}

Abstraction faite de quelques précurseurs, dont le premier et le plus célèbre reste ARISTOTE (343-340 av. J.C.), l'inventaire de l'ichtyofaune européenne a débuté au XVI ème siècle avec la parution quasiment simultanée de quatre œuvres majeures : BELON (1555) et 
RONDELET (1558) en France, GESSNER (1558) en Suisse, et SALVIANI (1554) en Italie (cf. notamment BLANCHARD, 1880 ; BAUCHOT, 1986 ; VASILIU et MANEA, 1987). Après cet extraordinaire tir groupé, les contributions suivantes furent plus espacées dans le temps, avant que le rythme ne s'accélère à nouveau avec l'émergence de la systématique linnéenne et des sociétés savantes, à l'aube du XIX ème siècle. Ce dernier sera très fertile en inventaires locaux de toutes sortes et de toutes qualités, notamment en France, à tel point qu'au début du XX ème siècle, la faune de France semble devoir être considérée comme définitivement décrite. De fait, l'importance des contributions naturalistes se raréfie alors au profit de l'ichtyologie économique (salmoniculture et aquaculture d'étang), au point que la parution de la dernière faune spécifiquement française par SPILLMANN en 1961 prend l'allure de point final pour l'ichtyologie naturaliste métropolitaine. Viendra alors une longue periode de léthargie avant que la dégradation du milieu et la modification des ichtyocénoses n'incitent à réexaminer plus attentivement nos peuplements et, en premier lieu, leurs structures taxonomiques, redonnant ainsi une dimension utilitaire aux inventaires. Cette évolution, grandement concomitante de celle de l'informatique (PERSAT, 1988), permet d'aller bien au-delà des simples compilations taxonomiques antérieures. Le précurseur de cette ère nouvelle est VERNEAUX, dont la thèse paraît dès 1973. En 1981, le Conseil Supérieur de la Pêche (CSP) et le CEMAGREF mettaient en place un protocole standard de recueil de données, mais ce n'est qu'en 1991 que l'association de ces organismes avec le Muséum National d'Histoire Naturelle permit la réalisation de l'Atlas préliminaire des poissons d'eau douce de France (ALLARDI et KEITH, 1991). Ensuite, progressivement, le CSP mit en place son réseau hydrobiologique et piscicole (RHP), et c'est finalement plus de 35 années après l'ouvrage de SPILLMANN que, à l'initiative de ALLARDI et KEITH, la réalisation d'un Atlas et d'une Faune de France réactualisés sera enfin entreprise.

Les introductions et le développement spectaculaire d'espèces nouvelles, telles que le silure (Silurus glanis), Pseudorasbora parva, le carassin doré (Carassius auratus), ou l'épyrine lippue, Pachychilon pictus (TALES et al., sous presse) pour ne citer que les plus récentes, sont l'une des raisons majeures d'une telle entreprise, ces espèces étant considérées comme plus ou moins nuisibles selon l'intérêt halieutique que leur portent les pêcheurs. Face à ces " envahisseurs ", il est de bon ton de militer en faveur de mesures " xénophobes " au nom de la défense du patrimoine biologique national, c'est-à-dire de nos poissons autochtones. Encore faudrait-il savoir donner un sens précis à cette dernière notion. En effet, la "satisfaction" de la communauté scientifique quant à son niveau de connaissance de la faune française suite au travail de SPILLMANN, cache en réalité pas mal de lacunes tant au niveau biogéographique que taxonomique. De fait, l'évaluation de notre réel patrimoine ichtyologique reste à faire. Le présent travail a pour objectif d'illustrer ce point de vue et de suggérer des directions d'investigations pour traiter les questions soulevées.

\section{DÉFINITION DU PROBLĖME}

\section{Qu'est-ce qu'un poisson autochtone?}

Si la France est une entité assez objective du point de vue géographie humaine, ce n'est certainement pas le cas du point de vue biogéographique et notamment ichtyologique. Trois des cinq principaux bassins français s'étendent hors de nos frontières, et chaque bassin est virtuellement indépendant, au moins à l'état naturel. Traiter le problème à l'échelle de la France n'a donc guère de sens: seule compte l'échelle du bassin. Ainsi, une espèce de la faune de France n'est pas forcément un poisson autochtone dans un bassin considéré, et n'y est à ce titre ni plus ni moins indésirable qu'un poisson « étranger ". Cela peut être aisément vérifié avec les espèces ayant vu leur répartition se modifier au cours du siècle écoulé : ainsi, le hotu, Chondrostoma nasus, espèce autochtone de France puisque citée dès le XVI ème siècle dans la Moselle (le achon de BELON, 1555), est un envahisseur récent dans les autres bassins français (NELVA-PASQUAL, 1985). 


\section{La répartition des poissons en France}

Examinée à l'échelle de nos principaux bassins hydrographiques, la faune ichtyologique française actuelle apparaît remarquablement uniforme.

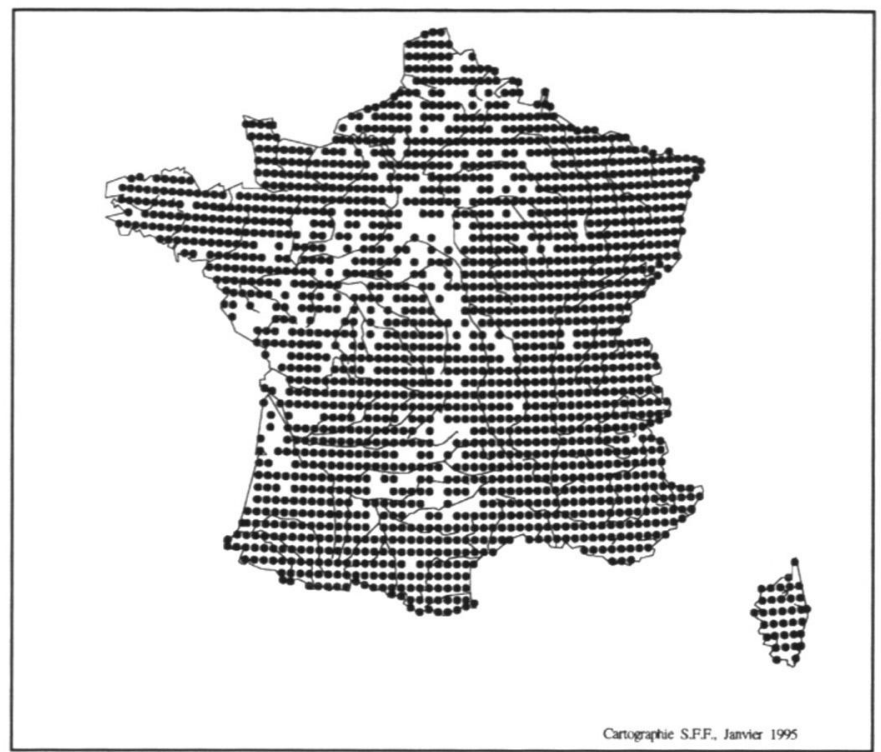

Figure 1

Carte des stations prospectées au 1er janvier 1995 pour l'Atlas des poissons d'eau douce de France, en cours de préparation.

\section{Figure 1}

Map of the prospected areas on January 1st, 1995 in the Atlas of the freshwater fishes in France, in progress.

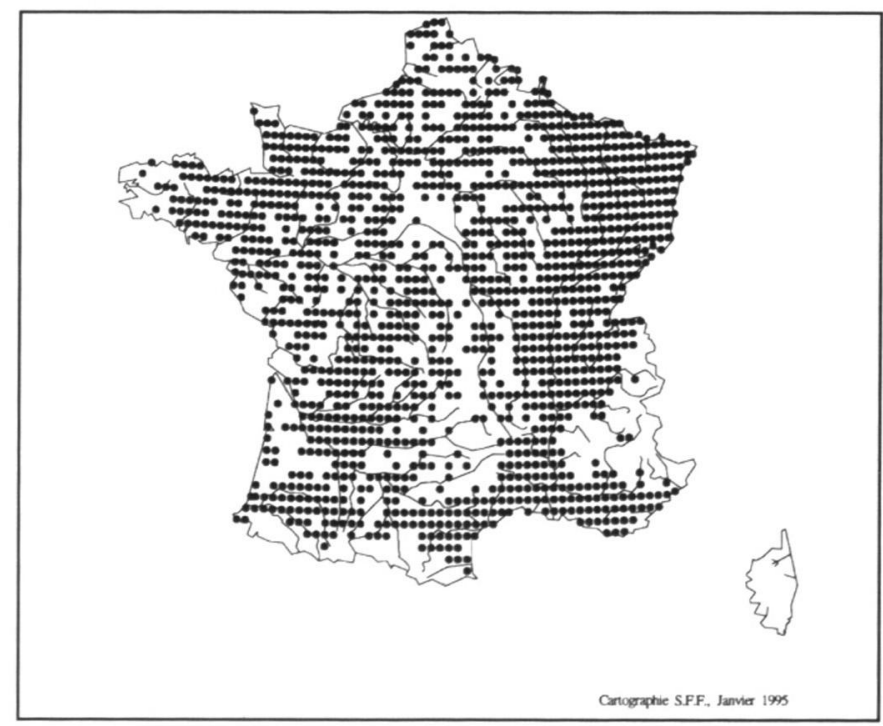

Figure 2

Aire de répartition d'une espèce " ubiquiste " en France : le cas du gardon Rutilus rutilus (extrait du même ouvrage).

Figure 2

Extension area of an " ubiquitous " species in France : the example of the roach Rutilus rutilus (drawn from the same work). 


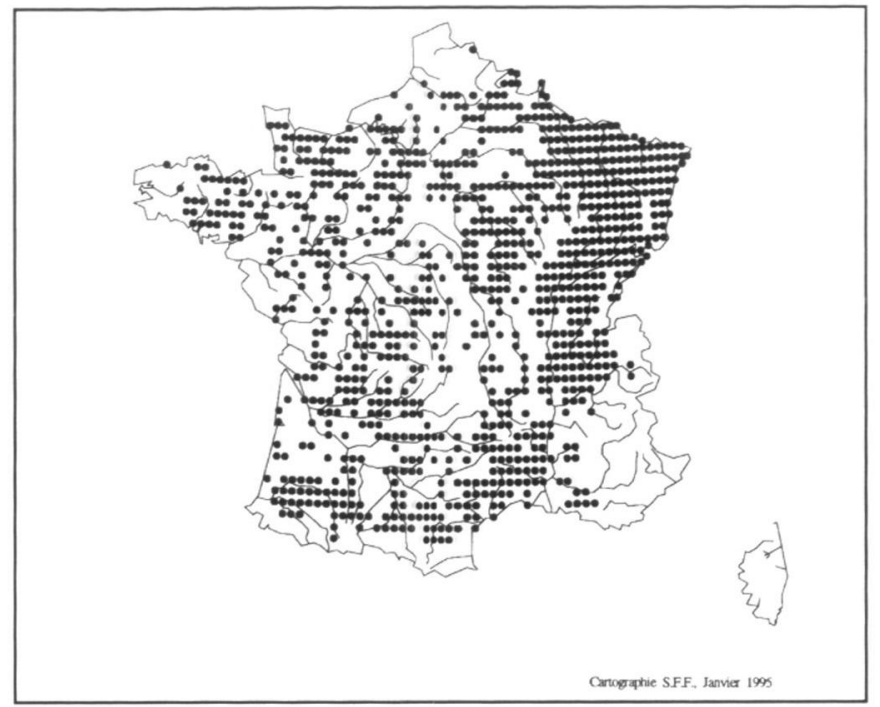

Figure 3

Aire de répartition d'une espèce "ubiquiste "à populations régionalisées : le cas de la vandoise Leuciscus leuciscus (base extraite du même ouvrage, avec illustration approximative des entités populationnelles évoquées dans la littérature ou observées par nous-mêmes ou nos correspondants).

\section{Figure 3}

Extension area of an " ubiquitous " species : the example of the dace Leuciscus leuciscus (drawn from the same work, but with an indication of the population units presumed according to the literature and our own or colleagues'observations).

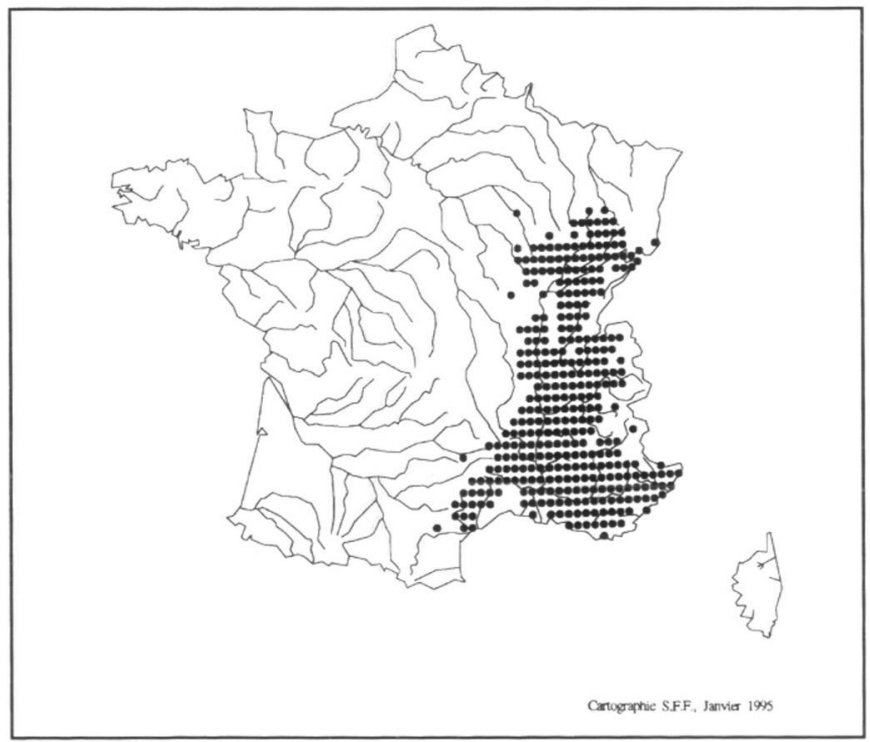

\section{Figure 4}

Aire de répartition d'une espèce hydrographiquement contrastée : le cas du blageon Leuciscus (Telestes) soufia (extrait du même ouvrage).

\section{Figure 4}

Extension area of an hydrographically contrasting species : the example of the blageon Leuciscus (Telestes) soufia (drawn from the same work). 
1 - Les principales espèces sont quasiment omniprésentes. La figure 1, qui en l'occurrence correspond à la carte des stations prospectées au 01/01/95 dans le cadre de l'Atlas des poissons d'eau douce de France en cours de réalisation, est pour l'essentiel la même que celle du gardon Rutilus rutilus (fig. 2), du barbeau commun Barbus barbus, des deux brèmes Blicca bjoerkna et Abramis brama, du brochet Esox lucius, de la carpe Cyprinus carpio, du chevaine Leuciscus cephalus, de la tanche Tinca tinca, de la perche Perca fluviatilis, du vairon Phoxinus phoxinus, ou de la vandoise Leuciscus leuciscus (fig. 3).

2 - Malgré cette large répartition, la présence de variantes locales traduisant l'ancienneté de l'isolement géographique des populations dans les différents bassins n'est guère évoquée que pour la vandoise (fig. 3) ou le blageon Telestes soufia (d'AUBENTON et al., 1971 ; GILLES et al., 1996).

3 - De même, relativement peu d'espèces présentent une répartition contrastée, avec présence massive dans un bassin et absence totale dans un autre (fig. 4).

4 - Enfin, et ce n'est pas la moindre des constatations, les bassins français n'hébergent que fort peu d'endémiques.

Cette situation contraste nettement avec celle de la péninsule ibérique où, sur une surface similaire à celle de la France, coexistent un nombre impressionnant d'espèces endémiques, chaque bassin possédant ses particularités faunistiques (ALMAÇA, 1978; DOADRIO et al., 1991).

Ces considérations posent le problème de l'origine de cette situation en France et, en conséquence, des moyens susceptibles d'être mis en oeuvre pour le résoudre. Pour cela, il convient de reconstituer l'histoire du peuplement ichtyologique des différents bassins français, afin de déterminer comment et quand une espèce donnée a pu atteindre un bassin donné. Cette démarche peut faire appel à des sources d'information très diverses.

\section{L'HISTOIRE, À DIFFÉRENTES ÉCHELLES}

Quatre périodes successives, d'ancienneté et de durée décroissantes, doivent être prises en compte pour la compréhension du peuplement ichtyologique français et, par extension, européen :

- la paléogéographie continentale et la paléobiogéographie au cours de l'ère tertiaire (de - 60 à - 2 millions d'années)

- la climatologie quaternaire et la dernière recolonisation postglaciaire (les 2 derniers millions d'années);

- la période historique (depuis l'antiquité) ;

- l'époque zoologique (du XVI ème siècle à nos jours).

A chacune de ces périodes peuvent être associés différents types d'informations biologiques, avec leurs avantages et leurs inconvénients respectifs.

- La paléontologie peut fournir des données précises quant à la présence ancienne d'une espèce ou d'une lignée, sous réserve que celle-ci ait bénéficié de conditions de fossilisation satisfaisante. Cela n'est que rarement le cas en eau douce et, généralement, seulement en milieu stagnant. Ainsi, c'est indubitablement en raison de son milieu vital que la tanche constitue la lignée de poissons d'eau douce la mieux référencée en Europe (OBRHELOVA, 1970; GAUDANT, 1980). Par contre, toute la cohorte des poissons rhéophiles est sousreprésentée, et ses composantes de petite taille quasiment absentes.

- L'archéologie peut également procurer de précieuses informations, à condition que les archéologues soient disposés à prêter attention à des fragments osseux aussi petits que les vertèbres, les dents ou les otolithes des poissons (STEINBERG, 1986), et que l'information 
susceptible d'être retirée des restes de poissons soit exploitée, ce qui n'a été que rarement le cas jusqu'à une époque récente. Aujourd'hui, grâce à la spécialisation poussée de quelques archéologues (DESSE et GARNIER, 1976; LE GALL, 1981, 1984), l'archéoichtyologie commence vraiment à être prise en compte dans les études paléoécologiques connexes à l'archéologie. Toutefois, les problèmes de conservation différentielle subsistent en raison, d'une part, de la nature (notamment la taille) des pièces osseuses susceptibles d'être conservées selon les espèces et, d'autre part, de la sélection certainement opérée par les pêcheurs de la préhistoire en fonction de la taille, de la qualité et de l'accessibilité des espèces. Enfin, les aptitudes de nos ancêtres dans le colportage de denrées alimentaires sur certaines distances ne doivent pas être sous-estimées : elles pourraient, par exemple, expliquer la présence de restes de saumon dans des sites situés sur le versant méditerranéen des Cévennes (CLEYETMERLE, 1990).

- Les écrits anciens, ou transcriptions de traditions orales, ou réminiscences linguistiques, par exemple dans les noms de lieux-dits (LE BERRE, 1970), peuvent également apporter des informations importantes, avec les précautions d'usage pour des références aussi particulières ;

- Enfin, les écrits modernes finalisés traitant des faunes locales sont évidemment les sources d'information les plus accessibles, mais ils sont susceptibles (au moins dans les premiers temps) de comporter des erreurs et surtout des omissions qu'il convient d'interpréter correctement.

Confronter toutes ces données aux connaissances actuelles dans le domaine de l'écologie des espèces est une opération indispensable pour corroborer ou infirmer toute information sujette à caution.

Enfin, les méthodes génétiques constituent désormais de puissants outils d'analyse qui pourront être judicieusement mis à contribution pour, certes, tester les différentes hypothèses susceptibles d'être émises quant à l'histoire des différentes espèces ou populations, quelles que soient les échelles de temps considérées, mais aussi pour achever l'inventaire zoologique de la faune de France qui pourrait encore révéler quelques surprises (par exemple, étude en cours sur la génétique des chabots).

\section{CHRONOLOGIE SUCCINCTE DU PEUPLEMENT ICHTYOLOGIQUE DE LA FRANCE ET PROBLÈMES AFFÉRENTS}

Sans entrer dans le détail de l'histoire biogéographique de l'ichtyofaune dulçaquicole française et donc européenne (BERG, 1949 ; THIENEMANN, 1950 ; BANARESCU, 1960, 1973, 1990), et en nous limitant aux espèces strictement d'eau douce (espèces primaires de MYERS, 1938), on peut distinguer un certain nombre d'événements déterminants :

- l'ouverture de l'Atlantique nord, détachant l'Europe de l'Amérique au cours de l'Eocène (POMEROL, 1973); la faune européenne reste néanmoins à fortes affinités nord-américaines ;

- le retrait de la mer de l'Oural dans la seconde moitié de l'Oligocène, qui assure la jonction entre l'Europe et l'Asie; l'Europe est alors envahie par la faune sibérienne, et notamment les cyprinidés;

- l'assèchement de la Méditerranée à la fin du Miocène (crise messinienne) (CITA, 1982), créant de nombreuses connexions entre les cours d'eau méditerranéens, y compris ceux d'Afrique du nord;

- les glaciations quaternaires, éliminant une grande part des faunes préexistantes, mais assurant nombre de connexions inter-bassins, directement par l'action des glaciers et indirectement par l'abaissement du niveau marin;

- la reconquête postglaciaire à partir des zones refuges méridionales (principalement bassin de la mer Noire par le Danube ; accessoirement bassin du golfe du Lion par le Rhône) ; 
- enfin, l'intervention de l'homme (colportage volontaire ou involontaire et création de connexions inter-bassins avec le creusement des canaux).

L'établissement des faunes originellement présentes dans les différents bassins européens, et donc français, doit tenir compte de chacun de ces événements. Ainsi, un genre ou une espèce présent à une époque donnée dans un bassin donné ne le sera pas forcément à l'époque suivante. C'est, par exemple, le cas du genre Silurus, présent dans la vallée du Rhône au Miocène (MEIN et al., 1983), mais indubitablement absent du même bassin à l'époque historique, avant sa réintroduction récente.

\section{PROBLÈMES RENCONTRÉS}

Une des principales difficultés réside dans l'évaluation de l'impact des glaciations quaternaires, et des possibilités de recolonisation durant les interglaciaires, le dernier étant celui que nous vivons présentement. Evaluer cet impact nécessite de connaître :

- ce qu'il y avait avant dans chaque bassin, chose évidemment fort délicate ;

- quelles espèces ont pu survivre sur place pendant les glaciations, ce que l'on peut évaluer d'après l'adéquation entre l'écologie des espèces actuelles et les reconstitutions climatiques ;

- quelles espèces ont pu recoloniser, en dehors de toute intervention humaine, les différents bassins après les glaciations, ce qui conduit à rechercher les traces des connexions hydrographiques récentes.

\section{Etat préglaciaire}

En ce qui concerne l'état zéro préglaciaire, nous ne disposons comme documents certifiés que des déterminations effectuées sur les fossiles (entre autres : GAUDANT, 1980, $1984,1987 \ldots$, ou OBRHELOVA, 1970, 1971, 1978...). Comme nous l'avons dit précédemment, les pièces sont rares, assez dispersées, et ne concernent qu'une fraction limitée des ichtyocénoses potentiellement présentes à chaque époque dans chaque bassin. Néanmoins, la présence de certaines espèces en certains sites permet d'émettre certaines conjectures quant à leur distribution ailleurs ou bien quant à celles d'autres espèces. Par exemple, la présence d'un genre d'aussi grande taille que Silurus dans la basse vallée du Rhône au Miocène implique la présence probable de tout un cortège d'espèces associées, de type potamique, et sans doute proches de celles vivant actuellement dans le bassin du Danube.

La position taxonomique de ces fossiles reste toutefois souvent imprécise, si bien que l'on ne sait si la présence préglaciaire d'une espèce dans un bassin donné peut être rapportée directement à la présence du taxon correspondant dans le même bassin après les glaciations, ou bien à une néo-colonisation à partir d'un bassin périphérique. II faut également tenir compte de la possibilité de persistance d'une espèce préglaciaire dans un bassin donné, avant éradication ou introgression postglaciaire par une espèce voisine. Ce fut peut-être le cas dans le bassin du Rhône, de façon naturelle, pour le genre Barbus, entre l'espèce méridionale $B$. meridionalis, "indigène ancien ", et le barbeau fluviatile $B$. barbus, "indigène plus récent " (PERSAT et BERREBI, 1990), et c'est apparemment le cas, mais de façon artificielle, pour le genre Chondrostoma, entre le toxostome $C$. toxostoma indigène et le hotu $C$. nasus introduit au siècle dernier. Dans ces exemples subsistent les traces de l'événement, mais le remplacement peut fort bien avoir été total si les niveaux taxonomiques étaient suffisamment proches. Ainsi, chez la truite commune, où existent deux races génétiquement distinctes, une atlantique et une méditerranéenne (GUYOMARD, 1989 ; POTEAUX et BERREBI, 1997), la forme méditerranéenne semblerait devoir être rapportée à une colonisation ancienne et la forme atlantique à une colonisation plus récente associée aux dernières glaciations, ce qui impliquerait alors une présence probable de la forme primitive méditerranéenne sur le versant atlantique avant son éradication par la nouvelle venue. 
Nous disposons, en revanche, d'informations cartographiques sur certaines connexions fluviales, telles celles établies au Pliocène entre le Danube, le Rhin, le Doubs et le Rhône (THIENEMANN, 1950 ; VILLINGER, 1986). On peut en inférer une grande homogénéité des ichtyocénoses en Europe moyenne, peu avant les glaciations. Toutefois, cela ne permet pas de préjuger de ce qui pouvait exister dans les bassins plus occidentaux (Seine, Loire, Garonne).

Une façon de contourner ces difficultés est d'essayer de voir ce qui, dans la distribution actuelle des espèces non manipulées, ne semble pouvoir être expliqué par des opportunités de recolonisation postglaciaires. Par exemple, l'omniprésence de la vandoise dans tous les grands bassins français, et notamment de nombreux bassins côtiers secondaires, milite fortement en faveur de sa préexistence à travers toute la France avant les glaciations (sauf, sans doute, dans les départements du Var et des Alpes-Maritimes).

Compte tenu des données disponibles, il est vraisemblable que le peuplement ichtyologique français était bien plus riche à la fin du tertiaire qu'il ne l'est actuellement.

\section{Impact des glaciations}

Pour comprendre l'impact des glaciations en Europe, et notamment en France, il suffit de rappeler par exemple la présence de traces de sols polygonaux, caractéristiques du permafrost ${ }^{\star}$, en Aquitaine ce qui correspond à des climats de type toundra sibérienne ou grand nord canadien. Sous de tels climats, le peuplement ichtyologique se réduit presqu'exclusivement aux salmonidés. Chaque glaciation a donc eu des effets très négatifs sur notre faune indigène. II semblerait, en outre, que la dernière phase glaciaire ait été la plus drastique pour la faune en général, et l'ichtyofaune en particulier. De fait, l'existence de certaines espèces dans certains bassins lors des interglaciaires précédents, telle que l'on peut l'établir par exemple à partir des citations archéologiques, ne garantit pas forcément leur persistance autochtone jusqu'à l'interglaciaire actuel. II convient alors d'évaluer leur aptitude à subsister dans le bassin considéré durant le maximum glaciaire. En effet, sauf existence d'une échappatoire suffisamment méridionale pour offrir des conditions thermiques point trop sévères, seules les espèces susceptibles de se reproduire à assez basses températures ont eu une chance de se maintenir. C'est le cas bien évidemment des salmonidés, mais aussi de la lote Lota lota, du chabot Cottus gobio, du brochet Esox lucius et, dans une moindre mesure, de certains cyprinidés rhéophiles, dont le plus cryophile est incontestablement la vandoise, avec des températures de reproduction aux alentours de $10^{\circ} \mathrm{C}$ (d'où, sans doute, la conservation des variantes locales chez cette espèce). En revanche, il apparaît difficile que les espèces potamiques, dont la moins thermophile est le gardon (reproduction aux alentours de $14^{\circ} \mathrm{C}$ ), aient pu survivre en dehors de l'extrême sud-ouest, du Languedoc-Roussillon ou de la Provence, le Rhône lui-même devant être aux époques glaciaires un fleuve épouvantablement froid et brutal (laves glaciaires), jusque et y compris son embouchure, au contraire de son delta actuel.

\section{récentes \\ Opportunités de recolonisations postglaciaires : les connexions fluviales naturelles}

L'évaluation des possibilités de recolonisation postglaciaire est une des clés nécessaires à la détermination du caractère autochtone ou non d'une espèce. Ces possibilités peuvent s'apprécier à travers la structure du réseau hydrographique. Pour le ou les derniers interglaciaires, des connexions tardives inter-bassins sont connues ou peuvent être envisagées au niveau de grands cours d'eau, de petits ruisseaux, ou bien de seuils hydrographiques peu marqués. En terme de migrations d'espèces, ces connexions peuvent avoir fonctionné à sens unique ou à double sens selon la nature des faunes en présence de part et d'autre, et la nature de la connexion. En effet, les captures de cours d'eau sont

\footnotetext{
*Permafrost : sol perpétuellement gelé des régions arctiques.
} 
toujours orientées et peuvent correspondre à des événements hydrauliques très courts (voire limités à une seule grande crue), lorsqu'elles reposent sur de fortes différences de niveaux entre bassins et des substratums plutôt meubles. En outre, leur efficacité en matière de transfert faunistique dépendra de la dimension des cours d'eau mis en jeu. Statistiquement, la quantité de captures est forcément bien plus grande sur les petits cours d'eau, de par leur nombre et leur position le plus souvent en limite de bassin. Les espèces de petits cours d'eau ont donc beaucoup plus d'opportunités de dispersion, mais il s'agit alors d'espèces apicales, généralement de petite taille et en nombre restreint (truite Salmo trutta, loches Nemacheilus barbatulus et Cobitis taenia, chabot Cottus gobio, vairon Phoxinus phoxinus, goujon Gobio gobio..., barbeau méridional Barbus meridionalis dans le midi). Par contre, dans le cas de captures de grands cours d'eau, certes bien moins fréquentes, c'est l'ensemble du peuplement d'un bassin, dont notamment les grandes espèces potamiques, qui peut profiter du passage.

L'énumération suivante donne un aperçu des connexions naturelles récentes connues, possibles, voire même encore potentiellement fonctionnelles (il reste encore beaucoup de cas à examiner), et des espèces éventuellement concernées :

- Danube -> Vistule -> Oder -> Elbe -> et au-delà :

- connexions inter-fluviales est-ouest entre grands cours d'eau de plaine le long des barrières morainiques de Pologne et de Prusse, ou à travers le lac Baltique, et permettant des migrations de faunes complètes (brèmes Abramis brama, Abramis ballerus et Blicca bjoerkna, carassin commun Carassius carassius, vimbe Vimba vimba, aspe Aspius aspius, ide Leuciscus idus, able Leucaspius delineatus, loche d'étang Misgurnus fossilis ?) ;

- captures de têtes de bassin danubiennes en direction de l'Elbe, permettant le passage de certaines espèces apicales.

- Danube -> Rhin :

- captures de têtes de bassin danubiennes dans le Bade-Würtemberg, en direction du lac de Constance et du Rhin ;

- captures de têtes de bassin danubiennes en direction du Neckar et du Main (hotu C. nasus, blageon T. soufia ? chabot (RIFFEL et SCHREIBER, 1995)) ;

- pertes actuelles du Danube en direction de la Wutach, affluent du Rhin, à travers des terrains karstiques (pourraient occasionnellement laisser passer des alevins d'espèces assez apicales, telles le hotu).

- Rhin -> Angleterre :

- connexion à travers la mer du Nord durant 3000 ans au début de l'interglaciaire actuel (migration de faunes complètes vers l'est Angleterre) ; la connexion s'étendait aux tributaires de la Manche (dont la Seine) durant le maximum glaciaire, mais n'était alors vraisemblablement exploitable que par les espèces cryophiles : vandoise, vairon, chabot, loches, lote?

- Rhin $\rightarrow$ Meuse $\rightarrow$ Seine :

- capture de l'Aire, affluent de la Meuse, par l'Aisne, affluent de l'Oise.

- Rhin -> Rhône :

- captures de tête de bassin à travers le Plateau suisse par la Venoge en direction du Léman et du Rhône (FATIO, 1890), exploitables par certaines espèces apicales (truite atlantique, omble chevalier Salvelinus alpinus, ombre commun Thymallus thymallus, corégone Coregonus lavaretus, cyprinidés rhéophiles, ablette Alburnus alburnus);

- captures de tête de bassin à travers le seuil de Franche-Comté (Largue ?), passage possible pour certaines espèces rhodaniennes en direction du Rhin (blageon?), et réciproquement ; 
- captures de ruisseaux de tête de bassin de la Moselle vers la Haute-Saône?

- Rhône $<->$ Languedoc :

- confluences inter-fluviales lors des glaciations par abaissement du niveau de la mer dans le golfe du Lion (échanges de faunes complètes);

- Rhône <-> Vistre <-> Vidourle :

- Plaine d'inondation ouest-camarguaise (espèces limnophiles);

- Vidourle <-> Hérault : cône de déjection de l'Argentesse, affluent du Vidourle, actif vers l'Hérault en temps de crue (espèces rhéophiles apicales, chabot, truite, blageon, barbeau méridional ?);

- Hérault <-> Orb <-> Aude <-> Pyrénées orientales : plaine d'inondation et étangs côtiers récents (quelques milliers d'années, tout au plus) du Languedoc (échanges de certaines espèces).

- Rhône -> Argens-Côte d'Azur :

- captures de têtes de bassin possibles à travers les karsts entre l'Arc et l'Argens, et entre le Verdon et le versant Côte d'Azur (espèces apicales : chabot, barbeau méridional, blageon, toxostome Chondrostoma toxostoma?).

- Aude $<->$ Garonne :

- succession de captures de têtes de bassin au quaternaire, du Fresquel (affluent de l'Aude) vers l'Agout (affluent du Tarn), et de l'Hers (affluent de la Garonne) vers l'Aude et, même anciennement, écoulement direct probable de l'Aude dans la Garonne (CAVAILLE et al., 1975), (échanges de faunes complètes : toxostome, barbeau méridional, blennie Blennius fluviatilis, vandoise, ablette, loche franche Nemacheilus barbatulus ?...).

- Catalogne espagnole $\rightarrow$ Roussillon : truite ?).

- captures de tête de bassin dans la région de Font-Romeu? (barbeau méridional,

- Loire -> Garonne, Loire -> Rhône :

- captures d'une tête de bassin de l'Allier en direction du Chassezac (affluent du Rhône) et du Lot (affluent de la Garonne) au niveau du causse de Montbel (Lozère) (espèces apicales : truite, vairon, loche franche, chabot, goujon ?) ;

- par ailleurs, la distribution géographique de deux espèces moins apicales, l'ombre commun et la lote, suggère l'existence d'une connexion Loire-Rhône de plus grande amplitude mais vraisemblablement beaucoup plus ancienne (PERSAT et al., 1978).

\section{Interférences humaines}

Sous ce titre sont regroupées toutes les interventions humaines non-répertoriées dans la littérature et qui peuvent masquer les distributions originelles de nos espèces. En l'occurrence, ce sont essentiellement des transplantations suffisamment anciennes pour ne pas avoir été mémorisées. Ce pourrait être aussi des éradications d'espèces particulièrement recherchées mais, en ce cas, leur valeur aurait probablement conduit à leur signalement dans des écrits.

En fait, sans vouloir faire abstraction d'opérations éventuellement plus anciennes mais forcément plus localisées, les deux périodes semblant les plus propices à une dispersion active mais non répertoriée sont l'antiquité romaine et le Moyen âge.

Les Romains sont bien connus à la fois pour leur propension à conserver des poissons dans des viviers, ce qui suppose une aptitude à les transférer vivants sur d'assez longues distances, et leurs aqueducs titanesques, qui impliquaient d'importants transferts d'eau courante à l'intérieur d'un bassin mais aussi entre bassins (cas célèbre de celui du Pont du 
Gard, amenant à Nîmes l'eau de la région d'Uzès, c'est-à-dire du bassin du Rhône vers le bassin du Vistre). Ils ont pu également construire des canaux de navigation : FATIO (1890) évoque un "canal d'Entre-Roches, que l'on dit avoir été établi par les Romains " entre le lac de Neuchâtel et le lac Léman.

Dans le même genre, Charlemagne souhaitait relier le Danube au Rhin et il semble bien qu'un éphémère canal ait été construit, même s'il n'a sans doute jamais été fonctionnel.

Le Moyen âge est bien connu pour l'importance civilisatrice des communautés religieuses. La place du poisson dans les rites de la chrétienté conduisait naturellement celle-ci à pratiquer la pêche et la pisciculture. La chaîne des grands lacs alpins, de l'Autriche à la Savoie, a fixé un grand nombre d'établissements religieux qui ont tous eu intérêt à développer la pêche et à colporter, de lac en lac, les espèces habituellement consommées.

Si l'on prend le cas du Léman, aucune espèce n'est susceptible d'y avoir survécu pendant la dernière glaciation, puisque ce n'était alors qu'un glacier. Après le retrait des glaces, les pertes du Rhône près de Bellegarde ont constitué un seuil infranchissable pour la plupart des espèces rhodaniennes susceptibles de recoloniser le lac depuis l'aval. Seules les espèces les plus rhéophiles (truite, vairon, ombre) ont, peut-être, pu passer (mais pas même la vandoise ou le blageon, PEDROLI et al., 1991). Sauf captures postglaciaires tardives à travers le plateau suisse, l'essentiel de la faune lémanique serait alors le fait de la main de l'homme, peut-être dès les romains (notamment avec la construction d'un canal entre les bassins de Neuchâtel et du Léman), ou durant le Moyen âge et la Renaissance (et encore actuellement, bien évidemment).

Dans le cas de transplantations par l'homme, les espèces les plus fréquemment manipulées devaient être les plus rustiques et/ou les plus appréciées : de fait, les espèces capables de vivre en étang ont certainement été les plus propagées. Par exemple, l'aire de répartition de la tanche en Europe ne correspond à aucune logique biogéographique et ne peut être expliquée que par des introductions systématiques. II en est certainement de même, à des degrés divers, pour la carpe Cyprinus carpio, le gardon, le rotengle Scardinius erythrophtalmus, les brèmes commune et bordelière, la perche Perca fluviatilis, voire le brochet, et le silure dans certains lacs suisses dont celui de Constance. On ne peut, en outre, exclure que le peuplement en ombles et corégones de certains grands lacs alpins soit également le fruit d'introductions très anciennes (BATIAS, 1958).

\section{CONCLUSIONS}

Les précédentes considérations portent à croire que la distribution actuelle de nombre de nos espèces " indigènes " dépasse de beaucoup leurs distributions naturelles initiales. Afin de redessiner celles-ci, et donc de mieux évaluer la valeur patrimoniale de chaque population dans chaque bassin, il est nécessaire de collecter le maximum d'informations par espèce et par bassin. Le réseau hydrographique de l'Europe occidentale peut être décomposé en un certain nombre d'entités plus ou moins indépendantes, dont il conviendrait d'établir les ichtyofaunes originelles (Tabl. l).

Le bassin de référence pour l'ichtyocénose européenne, et donc française, est celui du Danube. C'est le plus riche en espèces, et le principal vivier pour la recolonisation postglaciaire de l'Europe moyenne. A partir de celui-ci, deux voies de recolonisation sont possibles : l'une par le nord, caractérisée par les faunes de la Vistule et de l'Oder (bassin de la Baltique), et l'autre par l'ouest, caractérisée par les faunes rhénanes (bassin de la mer du Nord, et accessoirement de la Manche). Au-delà, vers l'ouest, différentes entités peuvent être définies en fonction de leur degré d'éloignement par rapport aux principaux axes fluviaux. Une proposition de partition est illustrée par la figure 5. Certains grands bassins ont été découpés en sous-bassins d'accessibilité ou de viabilité différentes selon les espèces. De même, les différentes unités ont été juxtaposées en fonction de leur probabilité de similitude faunistique. Cette partition est largement subjective et sera sujette à réajustement en fonction de la progression de nos connaissances. 


\section{Tableau I}

Proposition de liste et code (retenu pour le tableau II) des unités hydrographiques à considérer dans l'évaluation de la distribution biogéographique naturelle des espèces en France et dans les contrées avoisinantes.

\section{Table I}

List for the hydrographic units to be considered in the evaluation of the distribution of native freshwater fishes in France and surrounding districts, with their coding for the table II.

\begin{tabular}{|c|c|}
\hline \multirow{2}{*}{$\begin{array}{l}\text { Mer Noire } \\
\text { Danube }\end{array}$} & 1 \\
\hline & 10 \\
\hline \multicolumn{2}{|l|}{ Baltique } \\
\hline Vistule & 20 \\
\hline Oder & 21 \\
\hline Rhin-Mer du Nord & 3 \\
\hline Rhin & 30 \\
\hline Rhin alpin & 301 \\
\hline Bas-Rhin & 302 \\
\hline Main-Neckar & 303 \\
\hline Moselle & 304 \\
\hline Meuse & 305 \\
\hline Elbe & 31 \\
\hline Weser & 32 \\
\hline Escaut & 33 \\
\hline Est-Angleterre & 34 \\
\hline Seine-Manche & 4 \\
\hline Seine & 40 \\
\hline Basse-Normandie et Golfe Normando-Breton & 41 \\
\hline Haute-Normandie & 42 \\
\hline Somme & 43 \\
\hline Sud-Angleterre & 44 \\
\hline Nord-Bretagne & 45 \\
\hline Ouest-Bretagne & 46 \\
\hline Rhône-Méditerranée & 5 \\
\hline Rhône & 50 \\
\hline Bassin lémanique & 501 \\
\hline Bassin annecien & 502 \\
\hline Haut Doubs & 503 \\
\hline Saône-Doubs & 504 \\
\hline Hérault-Vidourle-Vistre & 51 \\
\hline Orb & 52 \\
\hline Aude & 53 \\
\hline Roussillon & 54 \\
\hline Catalogne & 55 \\
\hline Ebre & 56 \\
\hline Argens-Gapeau & 57 \\
\hline Var-Côte d'Azur & 58 \\
\hline Riviera & 59 \\
\hline Loire-Nord-Gascogne & 6 \\
\hline Loire & 60 \\
\hline Sud-Bretagne-Morbihan & 61 \\
\hline Vilaine & 62 \\
\hline Vendée & 63 \\
\hline Charente-Poitou & 64 \\
\hline Garonne-Sud-Gascogne & 7 \\
\hline Garonne & 70 \\
\hline Landes & 71 \\
\hline Adour & 72 \\
\hline Biscaye & 73 \\
\hline
\end{tabular}




\section{Tableau II}

Tableau synoptique préliminaire des connaissances et interrogations quant à la distribution des espèces européennes de poissons primaires (MYERS, 1938) dans les différentes unités hydrographiques considérées (à partir, entre autres, de ALLARDI et KEITH, 1991 ; ALMAÇA, 1978 ; BANARESCU, 1960, 1973, 1990 ; BERG, 1949 ; BUSCH et al., 1988 ; CAZEMIER, 1988 ; ELVIRA, 1995 ; KIENER, 1985 ; KIENER et OLLIER, 1970 ; KIENER et al., 1981 ; LELEK, 1980 ; LUSK, 1996 ; MAITLAND, 1972 ; MAITLAND et LYLE, 1996 ; PEDROLI et al., 1991 ; SPILLMANN, 1961 ; WHEELER, $1977 . .$. , et de la base de données GICIM du Muséum National d'Histoire Naturelle de Paris).

N.B. - Beaucoup des indications qui y sont rapportées sont sujettes à caution, et c'est l'objet du présent travail que d'interpeller la communauté scientifique et naturaliste sur chaque cas répertorié.

Table II

Preview of the table compiling the knowledge and questions about the European primary freshwater fish distribution in the different hydrographic units considered.

N.B. - Many of the proposed indications are questionable, but this is the aim of the present work to call upon the scientific and naturalistic community about each case indexed.

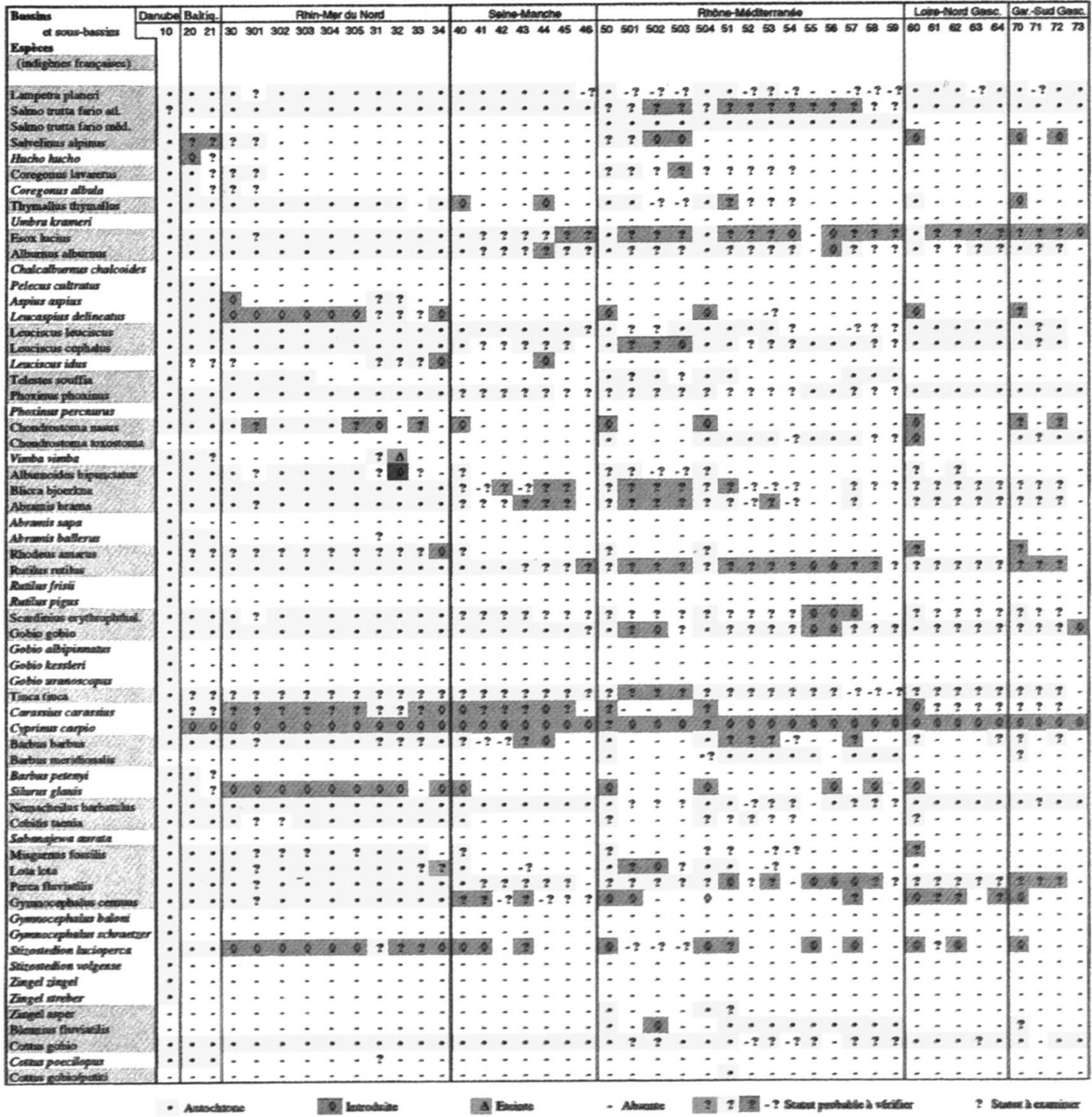




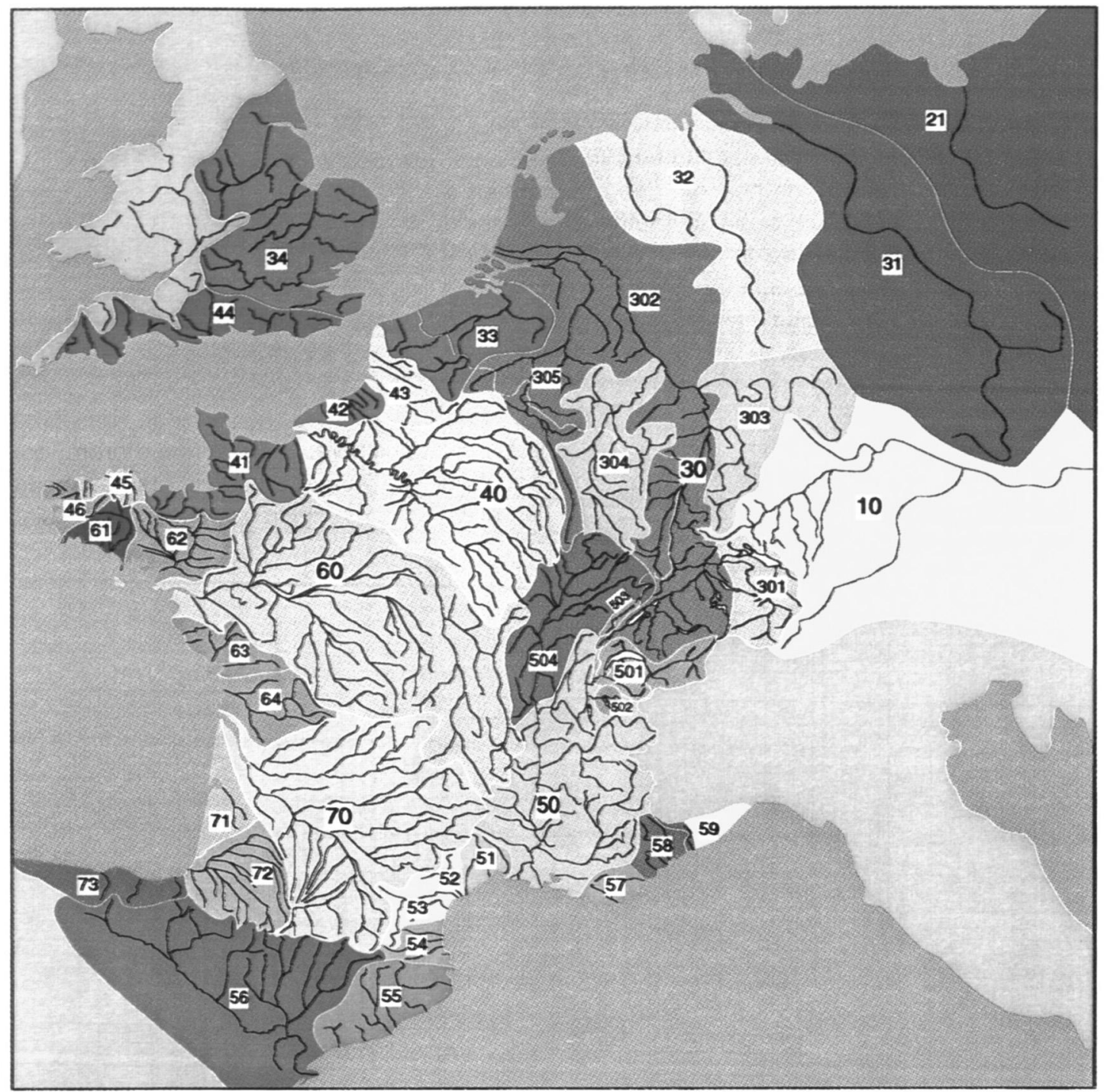

\section{Figure 5}

Carte des unités hydrographiques retenues dans le projet de reconstitution des aires biogéographiques originelles des différentes espèces de poissons en France et dans les contrées avoisinantes.

\section{Figure 5}

Map of the hydrographic units considered to rebuild the original distribution areas of freshwater fish species in France and surrounding districts.

Le tableau II donne une idée de l'ampleur du travail à accomplir. Chaque case représente le statut d'une espèce donnée (plus de 60) dans une unité donnée (plus de 40), selon une gamme de modalités allant de Absent à Autochtone, en passant par différents degrés de certitude. Si la réponse à beaucoup de ces cases ne pose guère de problèmes, il n'en est pas de même pour d'autres dont l'examen fera sans doute l'objet d'un long débat. Un statut provisoire est proposé d'après nos connaissances ou notre degré de réflexion actuels, afin de susciter les critiques et remarques de la communauté scientifique, et stimuler de nouvelles investigations.

Toute contribution est donc la bienvenue pour essayer de préciser ce qui dans nos ichtyofaunes françaises est autochtone ou ne l'est pas, car le peuplement qui doit servir de référence, notamment en matière de gestion et de réglementation des introductions, n'est pas complètement connu et dépend des bassins et de l'intervalle de temps pris en compte. 
Nous proposons qu'autochtone (ou indigène) ne soit pris qu'au sens strict d'espèce présente de façon naturelle dans un bassin donné, que cette présence soit très ancienne (préglaciaire, voire Pliocène ou même éventuellement Miocène dans certains cas) ou relativement récente (recolonisation naturelle postglaciaire). En revanche, les espèces qui ont profité d'opportunités offertes par l'homme (canaux) pour coloniser naturellement de nouveaux bassins ou sous-bassins, ne peuvent être considérées comme autochtones, mais seulement comme sub-spontanées. II apparaît toutefois souhaitable d'introduire des nuances dans le degré de xénobiontie. Xénobionte ${ }^{\star}$ de premier, deuxième ou énième ordre correspondrait au nombre d'étapes effectuées entre le bassin d'origine et le bassin colonisé. Par exemple, dans le cas d'espèces introduites, un transfert direct, quelle que soit la distance, donnera un xénobionte de premier ordre, alors qu'un transfert indirect (implantation dans un premier bassin, puis de ce premier bassin dans un autre) donnera un xénobionte de deuxième ordre. Pour une espèce sub-spontanée, le degré de xénobiontie correspondra au nombre de barrières hydrographiques franchies entre le bassin d'origine et celui considéré. Cette notion d'ordre, qui peut notamment avoir des implications au niveau génétique, ne permet toutefois pas de faire la différence entre des espèces allochtones vivant naturellement à proximité et des espèces des antipodes. C'est pourquoi nous suggérons d'utiliser le terme exogène exclusivement pour les espèces appartenant au même ensemble biogéographique, en l'occurrence ici l'Europe au sens large (sandre Stizostedion lucioperca, silure, etc.), et d'employer systématiquement le terme exotique pour les espèces appartenant à d'autres ensembles biogéographiques (Amérique du Nord, Extrême-Orient...) (perche-soleil Lepomis gibbosus, poisson-chat Ictalurus melas..., Pseudorasbora parva, carpe chinoise Ctenopharyngodon idella...). Ainsi, un exotique de premier ordre pourra fort bien côtoyer un exogène (éventuellement sub-spontané) de troisième ordre, sans que la nature fondamentalement différente de ces deux néo-implantés n'échappe au gestionnaire. S'il ne fait guère de doute qu'il faille à tout prix prohiber l'introduction d'exotiques, l'implantation de certains exogènes pourrait légitimement faire l'objet d'un débat, même s'il convient d'être très circonspect en la matière.

\section{BIBLIOGRAPHIE}

ALLARDI J., KEITH P., 1991. Atlas préliminaire des poissons d'eau douce de France. Coll. Patrimoines naturels, vol. 4, MNHN, SFF, CSP, CEMAGREF, Min. Env., 232 p.

ALMAÇA C., 1978. Répartition géographique des cyprinidés ibériques et secteurs ichtyogéographiques de la péninsule ibérique. Vest. Csl. Spol. Zool., 42, 241-248.

ARISTOTE, 343-340 av. J.C.. Histoire des animaux. Nouvelle traduction par J. TRICOT (1957). Bibliothèque des textes philosophiques, Lib. Philo. J. Varin, vol. 1 et 2.

AUBENTON (d') F., DAGET J., SPILLMANN C.J., 1971. Classification numérique des Blageons Leuciscus (Telestes) soufia (Pisces, Cyprinidae) (8ème note). Bull. Mus. Hist. Nat., 42 (5), 839-848.

BANARESCU P., 1960. Einige Fragen zur Herkunft und Verbreitung der Süsswasserfischfauna der europäisch-mediterranen Unterregion. Arch. Hydrobiol., 57, 16-134.

BANARESCU P., 1973. Some reconsiderations on the zoogeography of the Euro-Mediterranean freshwater fish fauna. Rev. Roum. Biol. Zool., 18, 257-264.

BANARESCU P., 1990. Zoogeography of freshwaters. Aula-Verlag, Wiesbaden, vol. 1, 511 p.

BATIAS A., 1958. Le lavaret du lac du Bourget (Coregonus lavaretus L.). Ann/s Sta. cent. Hydrobiol. appl., 7, 97-186.

\footnotetext{
* Xénobionte : élément étranger à un environnement donné.
} 
BAUCHOT M.L., 1986. Histoire des classifications des poissons. Océanis, 12 (3), 111-121.

BELON P., 1555. La nature et la diversité des poissons avec leurs pourtraicts représentés au plus près du naturel. Estienne, Paris, $448 p$.

BERG L.S., 1949. Freshwater fishes of the USSR and adjacent countries. Traduction de O. RONEN, 1965, Israël Progr. Scient. Transl., IPST Press, Jerusalem, vol. 1, 2 et 3 , $1510 \mathrm{p}$.

BLANCHARD E., 1880. Les poissons des eaux douces de la France. Baillière, Paris, $650 \mathrm{p}$.

BUSCH D., HAESLOOP U., SCHEFFEL H.J., SCHIEMER M., 1988. Fish and their environment in large European river ecosystems. The river Weser, FRG. Sci. Eau, 7 (1), 75-94.

CAVAILLE A., DEBAT P., CALAS G., 1975. Castelnaudary, notice explicative. Carte géologique de la France à 1/50 000, XXII-45, BRGM, Orléans, $17 p$.

CAZEMIER W.G., 1988. Fish and their environment in large European river ecosystems. The Dutch part of the river Rhine. Sci. Eau, 7 (1), 95-114.

CITA M.B., 1982. The Messinian salinity crisis in the Mediterranean : a review. Alpine Mediterranean Geodynamics Series, 7, 113-140.

CLEYET-MERLE, 1990. La préhistoire de la pêche. Collection des Hespérides. Errance, Paris, $195 \mathrm{p}$.

DESSE G., GARNIER J., 1976. Les Poissons. In « Préhistoire française ", vol. 1, 437-441, CNRS, Paris.

DOADRIO I., ELVIRA B., BERNAT Y., 1991. Peces continentales espanoles. Inventario y clasificacion de zonas fluviales. Coleccion Technica ICONA, Madrid.

ELVIRA B., 1995. Native and exotic freshwater fishes in Spanish river basins. Freshwat. Biol., 33 (1), 103-108.

FATIO V., 1890. Histoire des vertébrés de la Suisse - 5 : Histoire naturelle des poissons. Georg, Genève.

GAUDANT J., 1980. Mise au point sur l'ichtyofaune miocène d'Öhningen (Baden, Allemagne). C.R. Acad. Sci. Paris, 291, 1033-1036.

GAUDANT J., 1984. Nouvelles recherches sur les Cyprinidés (Poissons Téléostéens) oligocènes des Limagnes. Géobios, 17, 659-666, Lyon.

GAUDANT J., 1987. Mise au point sur l'ichtyofaune pliocène de Willershausen-am-Harz (Baden, Allemagne). C.R. Acad. Sci. Paris, 305, série II, 811-814.

GESSNER C., 1558. Historiae animalium liber IV. De piscium et aquatilium animantium natura. Zurich.

GILLES A., BARASCUD B., BOUCHARD P., CHAPPAZ R., 1996. Étude de la variabilité de Leuciscus soufia, poisson cyprinidé, par analyse du polymorphisme enzymatique et de caractères méristiques. C.R. Acad. Sci. Paris, Sci. Vie, 319, 393-399.

GUYOMARD R., 1989. Diversité génétique de la truite commune. Bull. Fr. Pêche Piscic., 314, 118-135.

KIENER A., 1985. Au fil de l'eau... en pays méditerranéen. Aubanel, Avignon, 307 p.

KIENER A., OLLIER J., 1970. Contribution à l'étude écologique et biologique de la rivière le Gapeau (Var). Hydrobiologia, 36 (2), 189-251.

KIENER A., DELIZE A., BELKIOR P., 1981. Aspects piscicoles du fleuve Argens (Var). Bull. Fr. Piscic., 282, 43-74. 
LE BERRE A., 1970. Ichthyonymie bretonne - 1 : Statistiques et systématique. Univ. Brest, $135 \mathrm{p}$.

LE GALL O., 1981. L'ichtyofaune dulçaquicole dans les gisements préhistoriques. Quaternaria, 23, $1-13$

LE GALL O., 1984. L'ichtyofaune d'eau douce dans les sites préhistoriques. Ostéologiepaléoécologie-palethnologie. Cah. Quat. CNRS, 8, 193 p.

LELEK A., 1980. Les poissons d'eau douce menacés en Europe. Conseil de l'Europe, Strasbourg, $276 \mathrm{p}$.

LUSK S., 1996. The status of the fish fauna in the Czech Republik. In KIRCHHOFER A. and HEFTI D., Conservation of endangered freshwater fishes in Europe, Birkhäuser, Basel, 89-98.

MAITLAND P.S., 1972. A key to the freshwater fishes of the British Isles with notes on their distribution and ecology. Freshwater Biological Association, scientific publication, 27, $139 \mathrm{p}$.

MAITLAND P.S., LYLE A.A., 1996. Threatened freshwater fishes of Great Britain. In KIRCHHOFER A. and HEFTI D., Conservation of endangered freshwater fishes in Europe, Birkhäuser, Basel, 9-21.

MEIN P., MÉON H., ROMAGGI J.P., SAMUEL E., 1983. La vie en Ardèche au Miocène supérieur d'après les documents trouvés dans la carrière de la montagne d'Andance. Nouv. Arch. Mus. Hist. nat. Lyon, suppl. 21, 37-44.

MYERS G.S., 1938. Freshwater fishes and West Indian zoogeography. Rep. Smithsonian Instn, 339-364.

NELVA-PASQUAL A., 1985. Biogéographie, démographie et écologie de Chondrostoma nasus nasus (L. 1758) (Hotu, poisson, téléostéen, cyprinidé). Thèse d'Etat, Univ. Lyon 1, 360 p.

OBRHELOVA N., 1970. Die Östeologie der Vorläufer von Tinca tinca (Pisces) aus dem Süsswassertertiär der CSSR. Abh. Staatl. Mus. Minerl. Geol. Dresden, 16, 99-209.

OBRHELOVA N., 1971. Vergleichende Östeologie der Gattung Leuciscus (Pisces) aus tertiären Schichten der nördlichen und westlichen CSSR. Paleontolog. Abhandl., 4 (A), 549-560.

OBRHELOVA N., 1978. Die Gattung Umbra Walbaum (Pisces) im nördböhmischen Tertiär, Entwicklungsgeschichte der Esocoidei Berg im Lichte der funktionalen Analyse. Sborn. Narodn. Muz. v. Praze, 34 (B), 119-171.

PEDROLI J.C., ZAUGG B., KIRCHHOFER A., 1991. Atlas de distribution des poissons et cyclostomes de Suisse. Documenta Faunistica Helvetiae 11, Centre suisse de cartographie de la faune, Neuchâtel, $206 \mathrm{p}$.

PERSAT H., 1988. De la biologie des populations de l'Ombre commun Thymallus thymallus (L. 1758) à la dynamique des communautés dans un hydrosystème fluvial aménagé, le Haut-Rhône français. Eléments pour un changement d'échelles. Thèse d'Etat, Univ. Lyon 1, $223 \mathrm{p}$.

PERSAT H., PATTEE E., ROUX A.L., 1978. Origine et caractéristiques de la distribution de l'Ombre commun, Thymallus thymallus (L. 1758), en Europe et en France. Verh. Ver. Angew. Limnol., 20, 2117-2121.

PERSAT H., BERREBI P., 1990. Relative ages of present populations of Barbus barbus and Barbus meridionalis (Cyprinidae) in southern France : preliminary considerations. Aquat. Living Resour., 3, 253-263.

POMEROL C., 1973. Stratigraphie et Paléogéographie. Ėre Cénozoïque (Tertiaire et Quaternaire), Doin, Paris, 269 p. 
POTEAUX C., BERREBI P., 1997. Intégrité génomique et repeuplements chez la truite commune du versant méditerranéen. Bull. Fr. Pêche Piscic., 344-345.

RIFFEL M., SCHREIBER A., 1995. Coarse-grained population structure in Central European sculpin (Cottus gobio L.) : secondary contact or ongoing genetic drift? J. Zoo. Syst. Evol. Research, 33, 173-184.

RONDELET G., 1558. L'histoire entière des poissons composée premièrement en latin, maintenant traduite en français avec leurs pourtraicts au naif. Macé Bonhomme, Lyon.

SALVIANI T., 1554. Aquatilium animalium historiae, liber primus, cum eorundem formis, aere excusis. Rome.

SPILLMANN C.J., 1961. Faune de France. 65 poissons d'eau douce. Lechevalier, Paris, 303 p.

STEINBERG M., 1986. Recherche d'une méthodologie appliquée à l'analyse des restes ichtyologiques du gisement de Lattes. Mém. Maîtrise, Univ. Sorbonne Paris I.

TALES E., KEITH P., GAYOU F., 1996. Première capture du cyprin lippu tacheté, Pachychilon pictus (Cyprinidae), espèce endémique d'Albanie, dans le bassin de la Garanne (France). Cybium (sous presse).

THIENEMANN A., 1950. Verbreitungsgeschichte der Süsswassertierwelt Europas. Die Binnengewässer 18, Stuttgart, $809 \mathrm{p}$.

VASILIU G.D., MANEA G.I., 1987. Istoria intiologiei romanesti. Bul. Cercet pisc., sup. 1, 332 p.

VERNEAUX J., 1973. Cours d'eau de Franche-Comté (massif du Jura). Recherches écologiques sur le réseau hydrographique du Doubs : Essai de biotypologie. Thèse d'Etat, Univ. Besançon, $257 \mathrm{p}$.

VILLINGER E., 1986. Untersuchungen zur Flussgeschichte von Aare-Donau/Alpenrhein und zur Entwicklung des Malm-Karsts in Südwestdeutschland. Jh. geol. Landesamt. Baden-Würtemberg, 28, 297-362.

WHEELER A., 1977. The origin and distribution of the freshwater fishes of the British Isles. J. Biogeog., 4, 1-24. 